\title{
Conflicting calculations of pelvic incidence and pelvic tilt secondary to transitional lumbosacral anatomy (lumbarization of S-1): case report
}

\author{
Charles H. Crawford III, MD, Steven D. Glassman, MD, Jeffrey L. Gum, MD, and \\ Leah Y. Carreon, MD, MSc \\ Norton Leatherman Spine Center, Louisville, Kentucky
}

\begin{abstract}
Advancements in the understanding of adult spinal deformity have led to a greater awareness of the role of the pelvis in maintaining sagittal balance and alignment. Pelvic incidence has emerged as a key radiographic measure and should closely match lumbar lordosis. As proper measurement of the pelvic incidence requires accurate identification of the S- 1 endplate, lumbosacral transitional anatomy may lead to errors. The purpose of this study is to demonstrate how lumbosacral transitional anatomy may lead to errors in the measurement of pelvic parameters. The current case highlights one of the potential complications that can be avoided with awareness.

The authors report the case of a 61-year-old man who had undergone prior lumbar surgeries and then presented with symptomatic lumbar stenosis and sagittal malalignment. Radiographs showed a lumbarized S-1. Prior numbering of the segments in previous surgical and radiology reports led to a pelvic incidence calculation of $61^{\circ}$. Corrected numbering of the segments using the lumbarized S-1 endplate led to a pelvic incidence calculation of $48^{\circ}$. Without recognition of the lumbosacral anatomy, overcorrection of the lumbar lordosis might have led to negative sagittal balance and the propensity to develop proximal junction failure. This case illustrates that improper identification of lumbosacral transitional anatomy may lead to errors that could affect clinical outcome. Awareness of this potential error may help improve patient outcomes.
\end{abstract}

http://thejns.org/doi/abs/10.3171/2016.5.SPINE16196

KEY WORDS sagittal alignment; pelvic parameters; lumbosacral radiographic measurements; anatomy; lumbar

$\Lambda$ DVANCEMENTS in the understanding of adult spinal deformity have led to an increased focus on sagittal alignment. ${ }^{4}$ Pelvic incidence has emerged as a key radiographic measurement that should closely match ideal lumbar lordosis., ${ }^{3,7}$ As proper measurement of the pelvic incidence requires accurate identification of the S-1 endplate, lumbosacral transitional anatomy ${ }^{1}$ may lead to errors. The current case highlights one of the potential complications that can be avoided with awareness.

\section{Case Report}

This 61-year-old man presented with symptomatic lumbar stenosis and sagittal malalignment. He had undergone prior lumbar surgeries at an outside institution.
The medical records listed the most recent surgery as a laminectomy and instrumented fusion from the third lumbar vertebra (L-3) to the fourth (L-4). His history revealed improvement after prior surgeries but recently progressive symptoms of lower back and leg pain consistent with neurogenic claudication. Increased pain with prolonged standing and walking were major complaints.

On examination, his lumbar incisions were found to be well healed with no signs of infection. His lumbar area demonstrated a loss of lordosis, and he stood with positive sagittal balance. Neurologically, he had good strength and sensation in his lower extremities. His reflexes were normal. Preoperative evaluation included a lumbar CT myelogram (Fig. 1). The segments were labeled by the ra- 


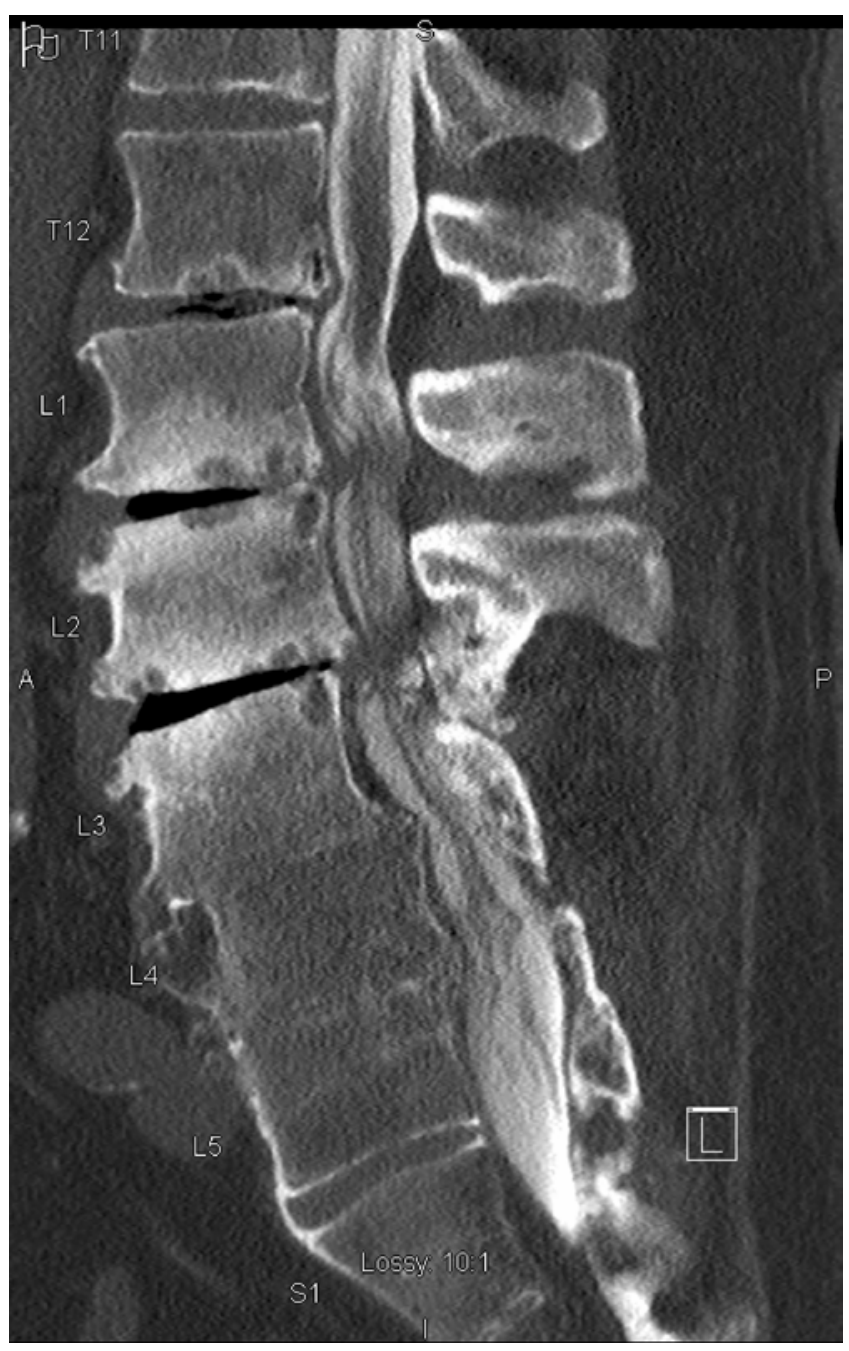

FIG. 1. Preoperative CT myelogram with radiologist's numbering of lumbar segments. The patient had previously undergone laminectomy and fusion of what is labeled L3-S1. Significant central canal stenosis and vacuum discs can be seen at the levels that are labeled T12-L3.

diologist consistent with the prior operative reports. Preoperative standing radiographs were initially measured using the S-1 endplate that correlated with the medical records. This endplate also correlated with the first nonrectangular vertebral body. Using this endplate for "S-1," the pelvic incidence was calculated to be $61^{\circ}$. Considering the thoracolumbar compensation, the "lumbar lordosis" was calculated as $27^{\circ}$ to the endplate of T-12 (Fig. 2A). As the thoracolumbar junction in the "normal spine" is straight, surgeons may choose to focus on any of the endplates from T-10 to L-2 when formulating a preoperative plan for restoring "normal" sagittal alignment. The preoperative measurements suggested that surgical correction would require an increase in "lumbar lordosis" of $34^{\circ}$. Additional review of the imaging studies during surgical planning identified a transverse process (no rib) at what was labeled T-12 (Fig. 2B). The patient was then considered to have 6 lumbar vertebrae or a lumbarized (lumbar shaped) S-1.

Remeasurement of the pelvic incidence using the newly identified S-1 endplate showed a lower pelvic incidence
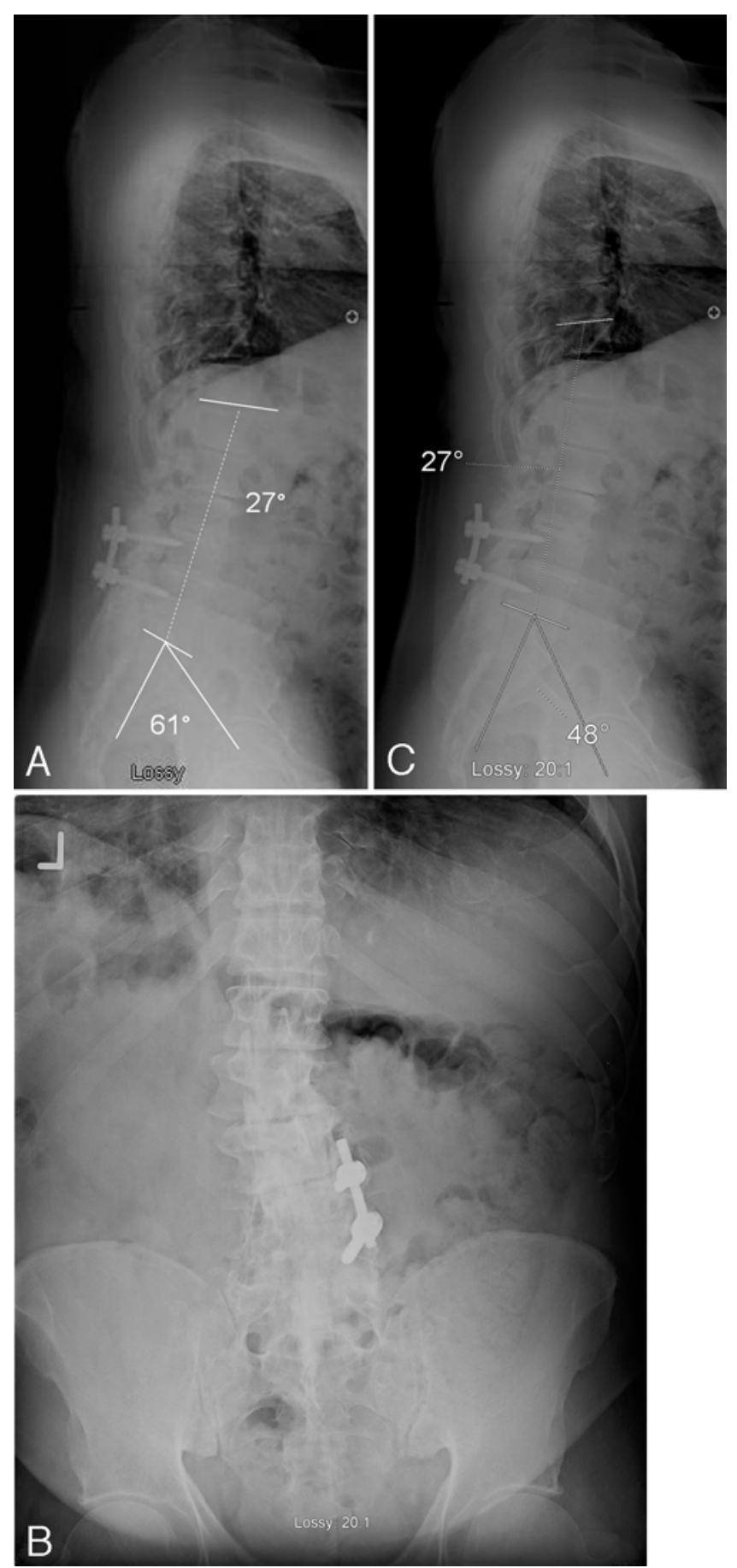

FIG. 2. A: Preoperative standing lateral radiograph showing the lumbar lordosis $\left(27^{\circ}\right)$ and pelvic incidence $\left(61^{\circ}\right)$ measured from the previously labeled S-1 superior endplate. Based on these measurements, the lumbar lordosis should be corrected approximately $30^{\circ}$ to match the pelvic incidence. B: Preoperative posteroanterior standing radiograph showing a transverse process and no rib at what was labeled T-12. C: Preoperative standing lateral radiograph showing lumbar lordosis $\left(27^{\circ}\right)$ and pelvic incidence $\left(48^{\circ}\right)$ measured from the "true" S-1 superior endplate. Based on these measurements, the lumbar lordosis should be corrected approximately $21^{\circ}$ to match the pelvic incidence. 


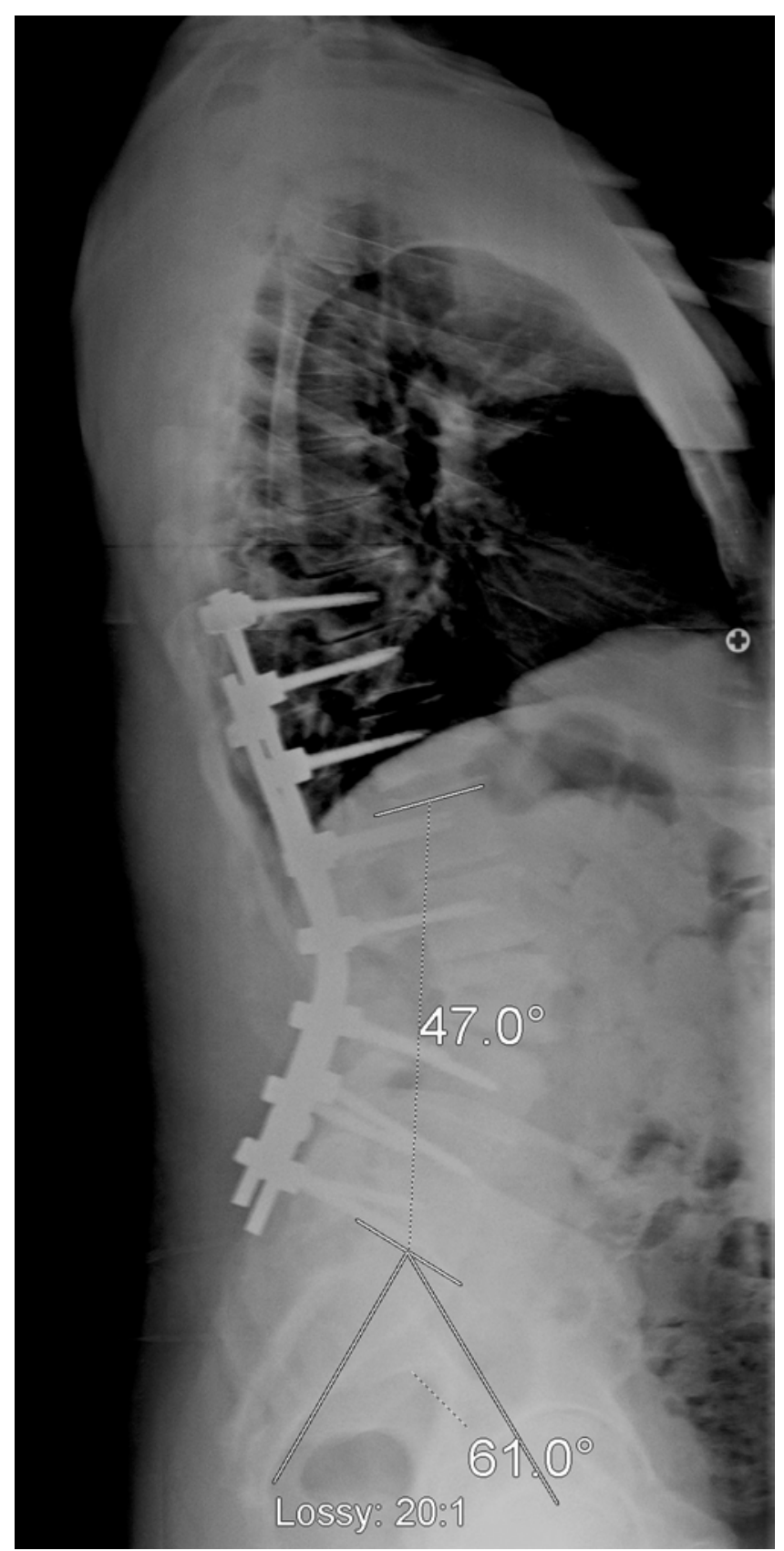

FIG. 3. Postoperative radiographic measurements on standing lateral radiograph using the previously numbered S-1 and showing a lumbar lordosis to pelvic incidence mismatch and high pelvic tilt $\left(30^{\circ}\right)$, although the sagittal vertical axis and sacral slope $\left(30^{\circ}\right)$ are within normal limits.

of $48^{\circ}$ (Fig. 2C). Based on the preoperative symptoms of standing and walking intolerance with claudication and positive sagittal balance and imaging findings suggesting that a decompression alone would be inadequate, the patient underwent a posterior laminectomy to decompress the spinal stenosis, a pedicle subtraction osteotomy of L-3 to add the needed $20^{\circ}$ of lumbar lordosis, and an instrumented fusion T10-S1 to maintain spinal stability and alignment. The secondarily identified "S-1 endplate" was suggested to provide the true pelvic incidence by postop-

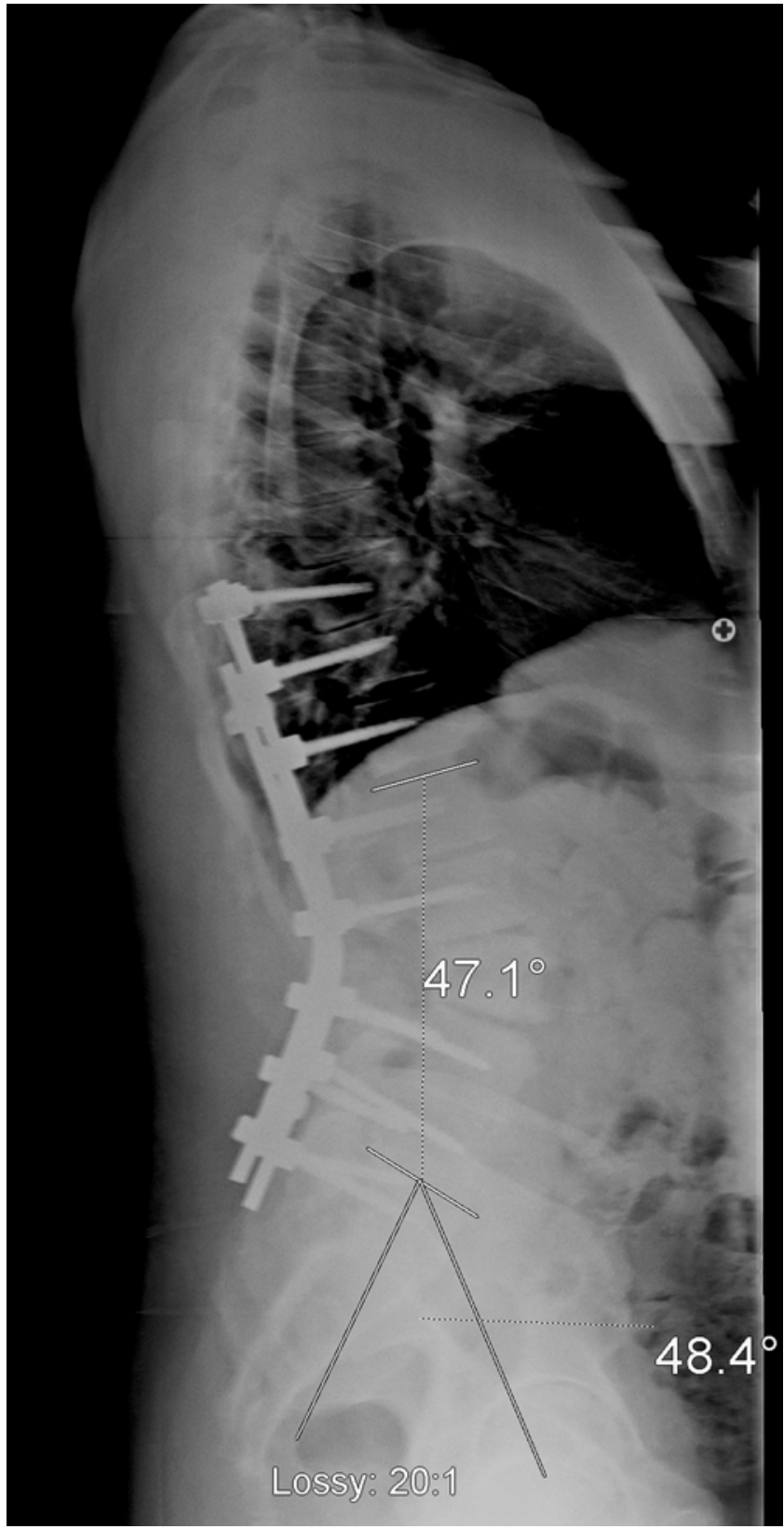

FIG. 4. Measurement of the "true" pelvic incidence on the same radiograph as in Fig. 3 showing a good match between lumbar lordosis and pelvic incidence. The sagittal vertical axis, sacral slope $\left(30^{\circ}\right)$, and pelvic tilt $\left(17^{\circ}\right)$ are within normal limits.

erative imaging (Figs. 3 and 4). Figure 3 shows the postoperative measurements with the initially identified S-1 endplate and a pelvic incidence to lumbar lordosis mismatch. The high pelvic tilt $\left(30^{\circ}\right)$ does not correlate with the normal sacral slope $\left(30^{\circ}\right)$ and normal sagittal vertical axis (C-7 plumb line). Figure 4 shows the postoperative measurements using "L-6" or the lumbarized S-1 endplate. The normal pelvic tilt $\left(17^{\circ}\right)$ correlates with the normal sacral slope $\left(30^{\circ}\right)$ and normal sagittal vertical axis (C-7 plumb line). Additionally in Fig. 4, the intercrestal line (the top of the iliac crest) appears to fall along the 


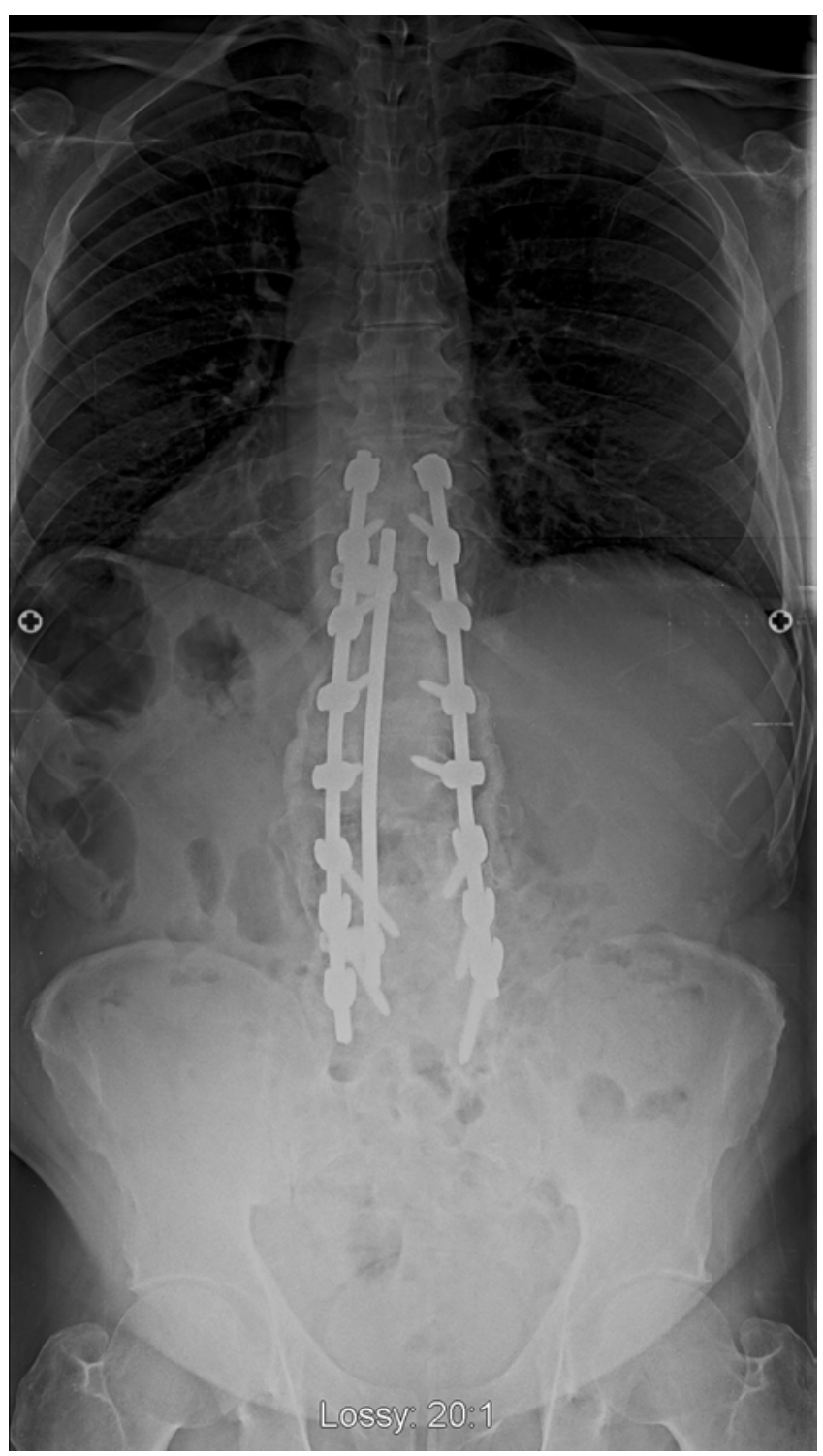

FIG. 5. Posteroanterior standing radiograph obtained approximately 18 months postoperatively showing a stable-appearing reconstruction and fusion. (Note the third rod stabilizing the osteotomy/laminectomy levels to minimize the risk of fatigue failure of the rod.)

"normal" L4-5 disc level, further supporting the measurements as shown.

Clinically, the patient was quite pleased with the outcome of surgery. He reported subjective improvement and demonstrated objective improvement in standing and walking tolerance. He had relief of his claudication symptoms and was able to stand erect with much less effort and energy expenditure. Radiographic follow-up demonstrated a stable reconstruction (Fig. 5).

\section{Discussion}

With an increased focus on the clinical importance of sagittal alignment ${ }^{5}$ and concurrent advancements in surgical techniques, ${ }^{2-4,7}$ the measurement of pelvic parameters (pelvic incidence and pelvic tilt) has become increasingly used to calculate the goals of surgical correction. ${ }^{2,3,7} \mathrm{Al}$ though overcorrection of sagittal alignment has been previously reported, ${ }^{3}$ no prior reports of errors due to lumbosacral transitional anatomy were identified in the literature or our personal experience. Overcorrection of lumbar lordosis has been implicated as a risk factor for the development of proximal junctional kyphosis or failure that may require revision surgery. ${ }^{1,6}$

The radiographic characteristics of transitional lumbosacral anatomy have been widely reported. ${ }^{5}$ Previously reported clinical relevance includes increased rates of degeneration at supra-adjacent segments and the risk of improper level identification during decompression surgery. ${ }^{5}$ The current case illustrates another circumstance (i.e., the measurement of pelvic parameters in adult spinal deformity) where improper identification of lumbosacral transitional anatomy may lead to errors that could affect clinical outcome..$^{1-4,6,7}$ Awareness of this potential error (pelvic incidence miscalculation due to lumbarization of S-1) may help surgeons avoid it during the complex process of planning surgery for sagittal malalignment.

\section{References}

1. Annis P, Lawrence BD, Spiker WR, Zhang Y, Chen W, Daubs MD, et al: Predictive factors for acute proximal junctional failure after adult deformity surgery with upper instrumented vertebrae in the thoracolumbar spine. Evid Based Spine Care J 5:160-162, 2014

2. Berjano P, Langella F, Ismael MF, Damilano M, Scopetta S, Lamartina C: Successful correction of sagittal imbalance can be calculated on the basis of pelvic incidence and age. Eur Spine J 23 (Suppl 6):587-596, 2014

3. Blondel B, Schwab F, Bess S, Ames C, Mummaneni PV, Hart $\mathrm{R}$, et al: Posterior global malalignment after osteotomy for sagittal plane deformity: it happens and here is why. Spine (Phila Pa 1976) 38:E394-E401, 2013

4. Glassman SD, Berven S, Bridwell K, Horton W, Dimar JR: Correlation of radiographic parameters and clinical symptoms in adult scoliosis. Spine (Phila Pa 1976) 30:682-688, 2005

5. Konin GP, Walz DM: Lumbosacral transitional vertebrae: classification, imaging findings, and clinical relevance. AJNR Am J Neuroradiol 31:1778-1786, 2010

6. Lau D, Clark AJ, Scheer JK, Daubs MD, Coe JD, Paonessa KJ, et al: Proximal junctional kyphosis and failure after spinal deformity surgery: a systematic review of the literature as a background to classification development. Spine (Phila Pa 1976) 39:2093-2102, 2014

7. Schwab F, Patel A, Ungar B, Farcy JP, Lafage V: Adult spinal deformity-postoperative standing imbalance: how much can you tolerate? An overview of key parameters in assessing alignment and planning corrective surgery. Spine (Phila Pa 1976) 35:2224-2231, 2010

\section{Disclosures}

Dr. Crawford reports a consultant relationship with Alphatec and Medtronic and being a course faculty member for Titan. Dr. Glassman reports a consultant relationship with Medtronic, being an employee of Norton Healthcare, and being a patent holder with and receiving royalties from Medtronic as well as being past president of the Scoliosis Research Society. Dr. Gum reports being an employee of North Healthcare; having a consultant relationship with Medtronic, DePuy, Alphatec, Stryker, Acuity, and 
PAKmed; receiving database support from NuVasive; receiving an honorarium from Pacira Pharmaceuticals; receiving a grant for travel funds from the Fischer Owen Fund; and receipt of funds by his institution from Integra, Intellirod Spine Inc., and ISSGF. Dr. Carreon reports an employee relationship with Norton Healthcare, a consultant relationship with AOSpine and Washington University School of Medicine; support of non-study related clinical or research efforts from OREF, Norton Healthcare, and SRS; and funds for travel from the Association for Collaborative Spine Research and the University of Southern Denmark as well as being a member of the University of Louisville Institutional Review Board. Dr. Carreon also reports that funds were provided by $\mathrm{NuV}$ asive directly to a database company.

\section{Author Contributions}

Conception and design: Crawford. Acquisition of data: Carreon, Crawford, Gum. Analysis and interpretation of data: Crawford, Glassman, Gum. Drafting the article: Crawford. Critically revising the article: all authors. Reviewed submitted version of manuscript: all authors. Approved the final version of the manuscript on behalf of all authors: Carreon. Administrative/technical/material support: Carreon.

\section{Correspondence}

Leah Y. Carreon, Norton Leatherman Spine Center, 210 East Gray St., Ste. 900, Louisville, KY 40202. email: leah.carreon@ nortonhealthcare.org. 